\title{
Only 'Time' will 'Tell': Influence of temporality on the interpretation of narrative discourses
}

\author{
Debmalya Biswas
}

PhD Research Scholar, Centre for Linguistics, Jawaharlal Nehru University. ORCID: 0000-00021543-6769. Email: debmal33_Ilh@jnu.ac.in, debmalyabiswas.professional@gmail.com

\begin{abstract}
The notion of language has been broadly understood in different ways with respect to existing literatures revolving around form, meaning, sound \& context. Although overtly these understandings do try to integrate with the functionality of a complex organic system, they glaringly lack reference to the basis for its realization, i.e., time. Approaches to problematize the understanding of language have overlooked the issue of time. Temporality introduces a distinct fuzziness in qualitative and abstract expressions beyond just the action or the state. It is also evident in the context of names in a diachronic sense. A systematic exploration of this gap can lead us to a time-oriented understanding of the faculty of language.
\end{abstract}

Keywords: temporality, space, time indexation, interpretation, discourse, language, part-of-speech categories.

\section{Introduction}

Every expression or articulation, using a streamlined linguistic system, involves an event or a state (internal and external) that inevitably requires space (real or imaginary) for plausible cognizance. Now, from both homogenous and heterogenous perspectives, any linguistic system centres around a community-aligned mechanism/core to capture change (both patterned and cluttered), which provides impetus to our expressive needs. Changes (both tangible and intangible) take place over time, and without time, space is static. So, the very basis of realisation of any linguistic system is time. Now, the notion of language has been broadly understood in different ways with respect to existing literatures revolving around form, meaning, sound \& context. Although overtly these understandings do try to integrate with the functionality of a complex organic system, majority of these glaringly lack reference to the basis for its realization, i.e., time.

\section{Introducing the issue}

In order to contemplate more seriously on this issue of reference of time, Berkeley's (1949) Corpuscular Philosophy of Time followed by Zwart's (1973) perspective become critical to the ongoing argument. Berkeley's ideas were grounded in a relational philosophy of time. For him,

This Open Access article is published under a Creative Commons Attribution Non-Commercial 4.0 International License (http://creativecommons.org/licenses/by-nc/4.0/), which permits non-commercial re-use, distribution, and reproduction in any medium, provided the original work is properly cited. For citation use the DOI. For commercial re-use, please contact editor@rupkatha.com. 
time was inseparable from the changes that constituted it. In Berkeley's words, time "is nothing, abstracted from the succession of ideas in our minds" (1949, p. 83). Consequently, a language user's experience of time can hence be projected as a function of the highly dynamic configuration of his or her ideas (crystallised through the faculty of language),

$$
\mathrm{E}(\mathrm{t}) \approx \mathrm{f}\left[\begin{array}{l}
\mathrm{di}_{\mathrm{L}} \\
\mathrm{dt}
\end{array}\right] \quad \begin{aligned}
& \text { The experience of time } \mathrm{E}(\mathrm{t}) \text { is instantaneous and can be represented as a } \\
& \text { differential equation, where a time-derived function of an individual's } \\
& \text { ideas in language can be notionally projected. }
\end{aligned}
$$

and the language user's idea of time emerges out of, what Hynes clarifies as, "reflection on this ideational variety" (2005, p. 339) while referring to Ardley (1968), Tipton (1974), Pitcher (1977) and Grayling (1986):

$$
\mathrm{i}(\mathrm{t}) \approx \mathrm{f}\left(\int_{1}^{n} i d i\right)\left[\begin{array}{l}
\text { The idea of time is collective and can be represented as an integral } \\
\text { equation, where an integration across time-points is projected of the } \\
\text { ideational variety. }
\end{array}\right.
$$

Zwart's ideation runs parallel to this:

Events do not just have their places in time, like pieces of wood floating in a river, but events constitute time. There is no flow of time beside or beneath the flow of events, but the flow of time is nothing but the flow of events. Therefore, one should not compare events in the flow of time with objects floating in a river, but with the molecules of water, the river is composed of. As the passing molecules of water constitute the flowing river, so the passing of events, i.e., their occurrence, constitutes the flow of time. (1973, p. 133)

Approaches to problematize the understanding of expression of ideas and events through language, have overlooked the issue of time. Temporality introduces a distinct fuzziness in qualitative and abstract expressions beyond just the action or the state. This gap inadvertently leads to relativistic mismatches and incongruities in expectation of language users.

The philosophical debate over time rages broadly between the A-theorists and the Btheorists. The A-theorists project an ontological distinction with respect to the way we understand the present, the past and the future. For them, there is an inherent dynamism in the very realisation of time. Contrarily, the B-theorists do away with the tripartite array of the 'tenses', arguing instead, in favour of a subtler, perceptual, relativistic reading of actuality. According to their stance, temporal reality is a conglomerate of temporal associations, like 'earlier than', 'later than', 'simultaneous with', etc. whereby we locate events in a time-bound matrix. Temporal reality governs "the truth-value of temporal sentences" (Farkas, 2008, p. 269). However, the aspect of 'change' gets overlooked when just confining the perspective to that of the 'momentous' and not of the 'momentary'.

Now, the gap between the way grammatical categories are projected and the way they are mentally processed while analysing discourse is somewhat scabrous in the ongoing line of argumentation. With the exception of Chinese and few other languages (where adverbs have a role in time indexation; e.g., /le/ in Chinese), conventionally only the function of tense is perceived to index time on the verb, whereby the canonical form gets conjugated with tense and aspect 
3 | Only 'Time' will 'Tell': Influence of temporality on the interpretation of narrative discourses

markers following subject-verb agreement norms in the language. However, while processing temporal discourses, marked by different "informative levels" (Becker \& Egetenmeyer, 2018, p. 28), language users move beyond a generalised understanding (time unmarked) of lexical items and make judgments based on time-specific understandings (derivative of the factors influencing that particular lexical item at that particular time). According to Becker \& Egetenmeyer (2018), "tense choice is only one tool contributing to the complexity of the temporal structure in natural discourse. Additionally, not only grammatical and lexical aspect, but also further lexical items such as adverb and conjunctions...play an important role" (p. 29).

Therefore, in order to better understand how language users interpret discursive ideas, we need to explore how part of speech categories (specially adjectives and adverbs), beyond just the verb, index time. This paper is a part of an ongoing doctoral dissertation which analyses temporal structuring and its influence on interpretation with respect to narrative discourses (with both linear and non-linear progression of time) in the language Bangla.

\section{Broad understanding of the research problem}

The problem is primarily discursive with larger implications concerning the philosophy of language and how we cognize the faculty of language. To arrive at the issue, critical questions on space, time and their bearing on language (expression and perception) have been revisited from Augustine (397-400 AD; trans. 1963), Berkeley (1710), Kant (1781), Peirce (posthumously collated by Hartshone, 1935), Bergson (1959), McTaggart (1921), Whitrow (1961) and Van Fraassen (1970).

The intention here is not to go deep into a discussion of the tensed vs. tenseless truth conditions as it simply pushes into a different domain whereas the methodological and the conceptual focus of the question at hand is discourse analysis. The primary focus is to explore whether and how part of speech categories, beyond the conventionally theorised notion of the inflected state of the verb, index time. The most contemporary evidence of research being pursued in a closely related context comes from a consortium led by Prof. Martin Becker at the University of Cologne investigating the temporal structuring of discourse. The paper cites a 2018 publication (in Lingua) from the same consortium, titled "A prominence-based account of temporal discourse structure". A publication by Becker \& Donazzan (2017) puts forth a contrastive analysis of the interpretation and the distribution of French adverbs or and maintenant alongside the Italian adverb ora. The objective was to highlight the analogy between the temporal and the argumentative application of the three particular adverbs focused in the paper. A secondary objective was to cull out the differences in the use of ora in comparison to or and maintenant.

In this paper, the primary concern is to understand how at the level of interpretation, adjectives and adverbs index time (although there is no overt orthographic evidence or simply orthography is under-equipped to represent this), beyond the conventionally theorised notion of the inflected state of the verb. To do this while the ongoing doctoral work deals with several narrative discourses in Bangla, in this paper, keeping the constraint of word limit in mind, a very small sample of the collected data will be presented. Based on the idea of time indexation by grammatical categories beyond just the verb, we can also subsequently try to arrive at a time and experience-based understanding of the faculty of language. 


\section{Scope and objective}

The research targets problems at two levels: perception/understanding and orthography. To provide some clarity to the issue, a very small but significant part of the data will be presented in a later section of the paper with two temporal narrative discourses, one focusing on the Bangla adjective /bhalo/ (meaning 'good') and the other focusing on the Bangla adverb /bhalobeje/ (meaning 'passionately' or 'lovingly' or 'fondly'). The discourses were followed by a simple

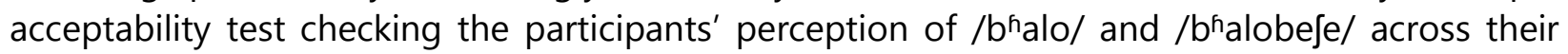
repetitions in the two narrative discourses with linear progression of time. At the level of perception, the findings gathered over 40 native Bangla speakers (non-linguists) provide encouraging evidence of time-specific readings being favoured over a time-independent or static reading.

The gap at the level of orthography is that there is no marker nor any overt semiotic cue in Bangla, for language users to readily grasp a temporal mismatch between or amongst repetitions of the same adjective or adverb, at different time points in a particular narrative discourse. Now, obviously, the gap in orthography is something immediately unresolvable but it can serve an explanatory purpose as one of the factors contributing to the language users' perceptual dilemma while attempting the acceptability test after reading the two narrative discourses.

There can be a further experimental modification whereby supporting statements, justifying the options given in the acceptability test, are not supplied. For the data presented in this paper, supporting statements were provided to overcome an experimental confound of participants not willing to judge the options carefully and asking too many questions which would interrupt the flow of the task. This confound can be tackled by having a very big pool of participants, which is what the ongoing doctoral work undertakes.

Now getting back to the level of perception, the paper has just presented findings for one

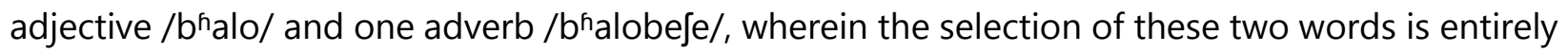
based on its remarkable frequency of usage and simplicity. Quite certainly, no conclusive statement can be made on the basis of just one adjective and one adverb. However, the immediate goal over here is not to make any radical statement. Rather, the plan is to highlight the feasibility of exploring this avenue, backed by the findings of this paper and the ongoing work (Becker et al.) probing the temporal structuring of discourse. The choice of the language, Bangla is due to the researcher's advantage of native intuition as well as its rich inventory of adjectives and adverbs. Additionally, a particular adjective or adverb can be used in several contexts and domains in Bangla. This facilitates the choice of adjectives and adverbs that can be looked at, across several contexts and domains.

Returning to the issue, language users are often unaware of the time-specific interpretations of adjectives and adverbs that are subconsciously made while analysing a temporal discourse. This research paper will be able to influence several subsequent independent papers on specific adjectives or adverbs and open the field for such investigations in other South-East Asian languages. 
5 | Only 'Time' will 'Tell': Influence of temporality on the interpretation of narrative discourses

\section{Exploration of pertinent philosophical strands}

In order to better appreciate the issue of indexation of time and how different interpretations of the same expression are actually time-derivatives at the specific point of reference in the discourse, it is necessary to briefly look at some relevant strands of philosophical deliberation across history.

Going significantly back in history, Augustine's philosophy of time (397-400 AD) revolved around his quest to arrive at a definition of time. Although he could tinker, flawlessly and effortlessly, with temporal terms (e.g., now, meanwhile, then, etc.) while using the faculty of language, Augustine was unsure about what the terms actually referred to. Some of the questions that had been troubling Augustine, are:

- Is time an aspect of the objective physical realm? If not, then, is time a subjective phenomenon?

- Are temporal relations, relations connecting physical events? If not, then, are they relations connecting personal mental events?

The answers to these queries were not found through a prosaic use of language. To add to his quandary, appropriate but prosaic use of language is in accordance with temporal terms, whereby at times there is a reference to the physical realm (objective), and at other times there is a reference to the experiential realm (subjective). Furthermore, Augustine was curious about:

whether temporal discourse requires reference to specifically temporal entities, i.e., entities or moments which exist independently of things, or whether time is adequately accounted for in terms of the temporal relations among events; whether the measurement of time depends upon the measurement of specifically temporal objects, or whether it is accounted for in terms of features of physical processes. (Lacey, 1968, pp. 219-220)

To support his argument, Augustine resorts to six fundamental premises, of which two are pertinent to the targeted issue:

A. "Only the present exists", i.e., "only that which is contained in the present exists, or only present things exist, i.e. past and future times, past and future things do not exist."

B. "The present has no temporal extension." (Lacey, 1968, pp. 220-221)

Now understanding these premises and being able to connect them with Augustine's concerns, will require an explication of what he implies by the present. In order to elucidate this further, a quick plunge into the stream of differential calculus is imperative. Differential calculus was devised much later by Newton (Methodus Fluxionum et Serierum Infinitarum, 1671) and Leibniz (1675), but there lies an intimate connection between its foundational principles and the way Augustine perceived the present. The operation of differentiation involves the calculation of derivative with respect to a specific variable and thereby it is possible to determine the exact or the instantaneous state at any particular point on the variable axis. For the present discussion, the relevant differential expression would be $\mathrm{d} x / \mathrm{d} t$, which signifies the rate of change of $x$ with respect to $t$ (time), making the instantaneous calculation of $x$ possible at any point of time $\left(t_{1} / t_{2} / t_{3} \ldots t_{n}\right)$ on the time axis. Differentiation as an operation is indispensible when change of $x$ is non-uniform. 
In the light of this much later development, Augustine's proposition of the present entails such an instantaneous consideration of ideas and events. "Only the present exists" implies that only an instantaneous interpretation of ideas and events holds validity at a specific point of time, where past interpretations and possible future interpretations do not coincide and therefore cannot compete for validity. This instantaneous perspective gets reinforced by Augustine's own impression:

If anything can be meant by a point of time so small that it cannot be divided into even the most minute particles of moments, that is the only time that can be called 'present'. And such a time must fly by so rapidly from future to past that it has no duration and no extension. For if it does have any extension, it can be divided into past and future; whereas the present does not take up any space. (1963, ch. 15)

It literally translates into the mathematical notion of limit. $\lim _{t \rightarrow 0} f(t)$ takes into account an interval of time that is almost 0 but not 0 , i.e., the minimum indivisible quantum. It is at this moment of time, that the instantaneous is the present.

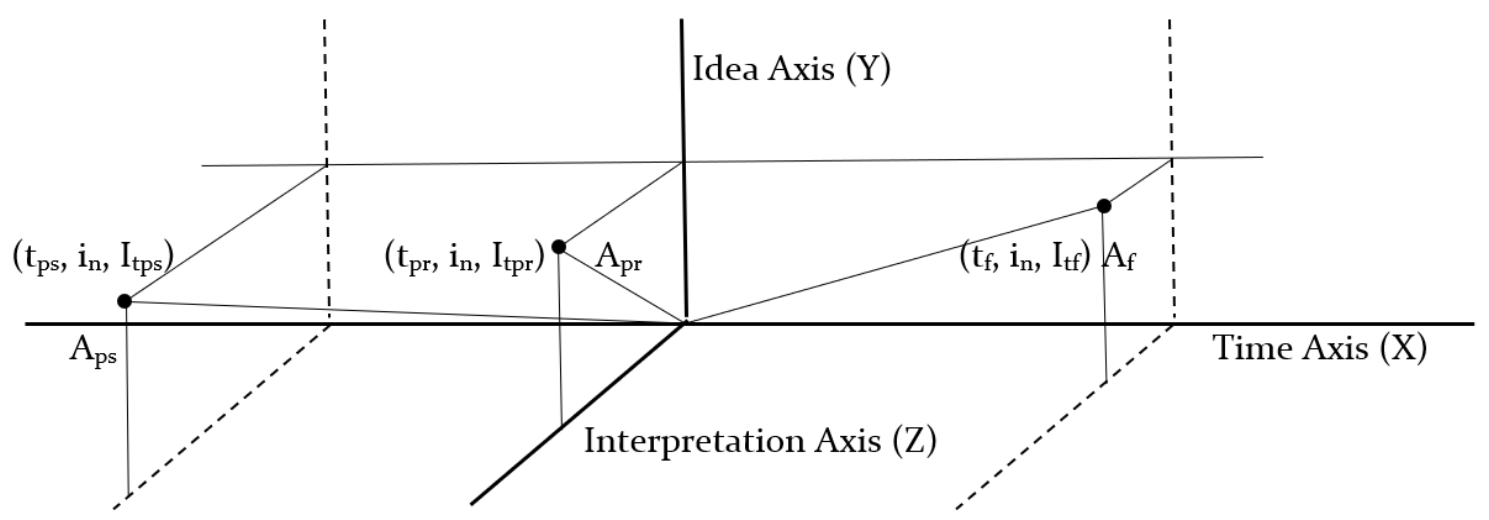

Figure 1: The time-idea-interpretation plot collected after anonymising participants as per their consent

$t_{p s}, t_{p r}$ and $t_{f}$ represent past, present and future co-ordinates on the time-axis (taken as $X$-axis)

$i_{n}$ represents the idea/event on the idea-axis (taken as $\mathrm{Y}$-axis) and $i_{n}$ remains constant across time $\mathrm{I}_{\mathrm{tps}} \mathrm{I}_{\mathrm{tpr}}, \mathrm{I}_{\mathrm{tf}}$ represent the specific interpretations on the interpretation-axis (taken as Z-axis) at time points $t_{p s}, t_{p r}$ and $t_{f}$ of the idea $i_{n}$

According to Augustine's first premise, only $A_{p r}\left(t_{p r}, i_{n}, I_{t p r}\right)$ "exists", as it indexes the instantaneous interpretation $I_{\text {tpr }}$ of idea $i_{n}$, valid only at the point of time, $t_{p r}$ which is the minimum indivisible quantum. So, if we try to visualise the timeline in terms of the above three-dimensional frame in microscopic detail, it would look something like this from an Augustinian perspective:

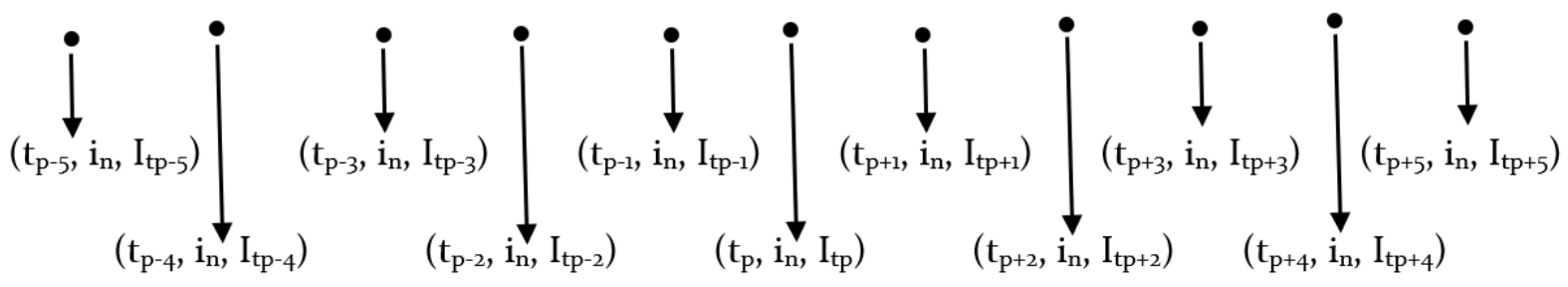

Figure 2: Simplifying the time-idea-interpretation plot in line with Augustine's viewpoint 
It is simply implausible for $A_{p s}, A_{p r}, A_{f}$ to coincide as that would indicate a staticity of interpretations and a freezing of time. In fact, this line of argumentation serves as a viable build-up to Berkeley's corpuscular philosophy of time, which argues in favour of time being realizable only in the succession of ideas in the mind, which representationally would look like:

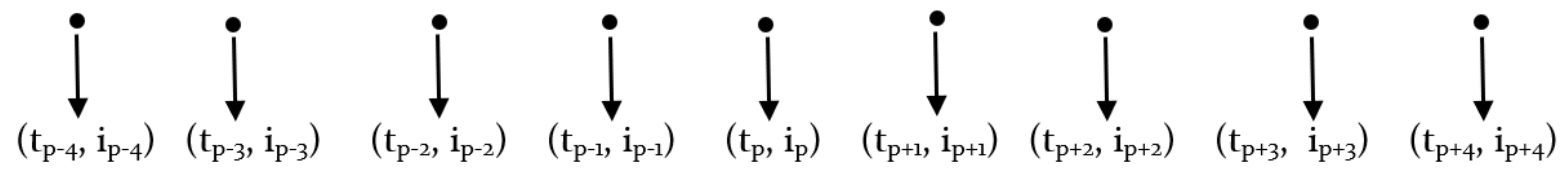

Figure 3: Plot of succession of ideas and flow of time as per Berkeleyian philosophy

Before moving ahead with Berkeley, it would be apt to highlight Augustine's view on the experiential dimension of time, which is further taken up by Berkeley:

It is in you, my mind, that I measure time. Do not interrupt me, or rather, do not allow yourself to be interrupted by the thronging of your impressions. It is in you, I say, that I measure time. As things pass by, they leave an impression in you; this impression remains after the things have gone into the past, and it is this impression which I measure in the present, not the things which, in their passage caused the impression. It is this impression that I measure when I measure time. Therefore, either this itself is time or else I do not measure time at all....it is not the future that is long, for the future does not exist; a long future is a long expectation of the future. Not is the past long, since it does not exist; a long past is a long memory of the past. (1963, Bk. XI, ch. 27, 28)

Berkeley (1710) believed time to be relational, while he also considered it to be uniform. His conventionalism is grounded in this uniformity. According to his philosophy of time, "the succession of ideas is constitutive of time, while parts of this succession are sufficiently stable and regular to serve as the basis for intersubjective temporal measures" (Hynes, 2005, p. 339). Berkeley dissected time to have a "corpuscularian structure" meticulously connected to Daniel Garber's (1982) terminology of "immaterialist corpuscularian" for Berkeley's stance on matter, whereby the "corpuscular substructure" of matter is sagaciously construed as "only a possible collection of regular ideas" (Hynes, 2005, p. 340). Correspondingly, Berkeley's critique of time, right down to the microscopic level, reveals its composition as "a regular succession of moments", where each moment is cognized as an individual perception, "a tempus minimum, within which no change occurs" (Hynes, 2005, p. 340). The Berkeleyian notion of time is conclusively deduced from an individual's perception of this very succession and the magnificent diversity of such regular moments, grounded in "the likeness principle": "an idea can be like nothing but an idea" (Berkeley, 1962, sec.8). The crux of Berkeley's argumentation is in a subjective understanding of time, whereby our experience of time is solely translatable as the movement of ideas in the mind. The pertinence of this philosophical stance for the paper is that if the term 'idea' is substituted by 'interpretation', then the corpuscular sense of time for a language user lies in the temporal matrix of interpretations, where each time-derived interpretation of an expression is irreducible, and adhering to "the likeness principle", each interpretation "can be like nothing but" an interpretation, which preserves its synergy with the expression/idea, of which it is an interpretation. The dynamic and unique nature of time-derived interpretations captures change and the process indexes the duration of time passed: 
Time therefore, being nothing, abstracted from the succession of ideas in our minds, it follows, that the duration of any finite spirit must be estimated, by the number of ideas or actions succeeding each other, in that same spirit or mind. (Berkeley, 1962, sec.98)

Furthermore, Berkeley advocates an individual perception, debunking a universal or homogeneous reading of time: "Each person's time being measured to him by his own ideas" (1944, p. 590). As Hynes aptly remarks on this,

[e]ach person's time is, therefore, private, that is time for me is the succession of ideas in my mind, and time for you is the succession of ideas in your mind. Time is, from the point of view of any given mind, unique to that mind and consists wholly in the succession of ideas it perceives. (2005, pp. 340-341)

It paves the way for an individual subjective interpretation of time realizable only inside the mind. Now, since time needs to be "abstracted from the succession of ideas" (Hynes, 2005, p. 339), therefore the faculty of language is inevitably responsible for capturing the succession of time since, as rational beings, we ideate or conceptualise in terms of the internalised language. This brings up the earlier stated objective, whereby it becomes vital to interrogate existing definitions and understandings of the faculty of language, in favour of a time-and-experience-based delineation.

The argument of subjective interpretation of time gets strengthened further when from a representationalist perspective, it is plausible that the human mind ideating through language, "can compare nothing but its own ideas" as the veil of perception always blocks gaining first-hand knowledge of reality and therefore, there cannot be any equivalence between "an idea and a mind-independent temporality" (Hynes, 2005, p. 339). Locke introduces a discursive angle to the issue, by arguing that language users process the idea of temporality from the succession of ideas, as if language users were to just stagnate on a single idea in the mind without any variation, it would not be possible to have any sense of change and consequently the duration/time over which this change is effected. Locke therefore emphasizes on time being "a kind of quantified change" and when there is a break in the succession of ideas, for instance when language users are in a state of sleep and incapable of conscious ideation, "the perception of time stops with it" (Hynes, 2005, p. 339). From a Lockean perspective, the representation of time can be considered to be discursive as it emerges "mediately in reflection" (Hynes, 2005, p. 339), i.e., while cognizing interpretations or ideas.

Kant further elaborates on these strands and takes the argument a step further by suggesting that human conception of time is not dependent on experience. To defend his stance, Kant reasons that an intuitive understanding of temporal terms or concepts like "before" and "after" would not be possible if time were not already a part of human knowledge, without the experience of it. This is what Kant ingeniously asserts as a priori, i.e., knowledge which is verified as true or false without experience and empirical validation. In the words of Kant:

Time is a necessary representation, lying at the foundation of all our intuitions. With regard to phenomena in general, we cannot think away time from them, and represent them to ourselves as out of and unconnected with time, but we can quite well represent to ourselves time void of phenomena. Time is therefore given a priori. In it alone is all reality 
9 | Only 'Time' will 'Tell': Influence of temporality on the interpretation of narrative discourses

of phenomena possible. These may all be annihilated in thought, but time itself, as the universal condition of their possibility, cannot be so annulled. (1998, Sec. II SS 5)

Experience of phenomena is absorbed through the human senses, resulting in an understanding which leads to that part of knowledge, dependent on experience and empirical justification, i.e., a posteriori, but the pre-requisite for this is the a prioripremise of time, on which all human intuitions are contingent: "neither coexistence nor succession would be perceived by us, if the representation of time did not exist as a foundation a priori." (Kant, 1998, Sec. II SS 5)

The essence emerges in the form of Kant's pristine inference that time is "a pure form of the sensuous intuition" (1998, Sec. II SS 5) indicating an "internal sense" (1998, Sec. II SS 7) without which the possibility of change cannot be grasped. Now, taking Kant's perspectives into account, in view of the topic of research, it can be catachrestically argued that a language user's sense of temporality in a discourse is something that exists a priori, whereas the differential (time-derived) interpretations hold true a posteriori, as each of such interpretations requires perceptual validation at a discrete indivisible moment (instantaneous) on the discourse timeline.

The subjective propositions of Augustine, Berkeley and Kant encounter opposition from Peirce, who asserted time to be a kind of objective order existing independent of any perceiving subject. Peirce placed his confidence in a real and not an ideal reading of time, positing it as "the way in which the conditions of objective possibility are displayed in the existing universe" (Helm, 1980, p. 379). Peirce treated existence as "to endure in time" or to be distinct in time "as an actual event" (Helm, 1980, p. 379). The distinctions between present, past and future according to Peirce can be explicated as: "The past is broken off from the future, and there is independence of the actual instant" (1974, vol.6, para 87).

"The present is a boundary" (1974, vol.7, para 536). Peirce's idea of the present acts as the border limiting the influence of the past. Contrary to Berkeley and Kant's proposition of succession, Peirce advocates discontinuity and there is a firm resolve in defending these breaks in time.

A certain tenet from Bergson's philosophical views also becomes relevant: "...our conception of matter is deformed by the space of our action, but...it is ultimately temporal" (Worms, 2005, p. 1230). Thinking is expounded as "a movement between "pure memory" ... and "perception" directed by the interests of our body in a present which itself implies an immediate synthesis of time" (Worms, 2005, p. 1230). "Pure memory" indicates the linguistic impression of an idea or expression, upon which a time-specific or instantaneous "perception" is built, governed by individual interests that condition a language user's interpretative faculty, at that particular discrete quantum of time.

McTaggart (1921) introduced the crucial dichotomy of the A and the B-series, problematizing the domain further. The issues that he addresses are that of denoting the symbiosis among past, present and future on one hand, and the connection between temporal precedence and simultaneity on the other. Fundamentally, the A-series covers a collection of positions which traverse the distant past, the near past, the present, the near future and the distant future. On the contrary, the B-series is grounded in temporal expressions, and moves smoothly from earlier to later positions. Concordances like 'earlier than', 'simultaneous with', 'later than' 
come under the purview of the B-series. McTaggart does not prejudice either, rather he suggests an undeniable dependency - a B-series is not realizable without an A-series. To explicate this, ideas, events or interpretations can be accorded precedence, simultaneity or succession (B-series) in language, only when they have already been placed on the discursive timeline, i.e., when they already have a position in terms of A-series. Since the 1950s, the conflict between the two opposing schools of thought, namely the A-theorists and the B-theorists has focused on the nature of temporal language and the cues that it can provide to better understand the nature of time, bringing us back to the idea of a time and space-oriented understanding of the faculty of language. The primary opposition to tense being the sole indexer of time, which is the stance in the A-series, comes from the B-theorists who argue that the truth-conditions, denoted in tensed sentences, are independent of tense or are tenseless. McTaggart's seminal contribution to the issue of language and temporality, makes him a cardinal figure whose mention becomes ineluctable.

Our primary focus is in a way linked to the opposition of the A-stance, as the paper aims to stir critical questions exploring how time is not just indexed by tense on the verb by looking at other part-of-speech categories in temporal discourses.

Whitrow in The Natural Philosophy of Time (1961) attempts an ambitious unification of the multitudes of strands which encompass the scholarly deliberations on how the idea of time penetrates thought and perception, intertwined with the faculty of language. Whitrow places immense importance on the immediacy of time (instantaneity of time) or on the flow of time as an integral part of our consciousness, which marks a subjective retracing of the Berkeleyian and the Kantian premise. Without going into the tensed vs. tenseless muddle, Whitrow opines that "there is public time, as part of the framework of the everyday world, and beyond this scientific time, as a further elaboration of commonsense public time" (Kneebone, 1964, p. 87).

Van Fraassen in An Introduction to the Philosophy of Time and Space (1970) explains the conception of "essential change" underlying the Aristotelian theory of duration. The generation of expressive needs and the way language-users use language can be roughly attributed to "essential change", to expound which Van Fraassen corroborates the notion of a family of properties, each property serving as a variable, the aggregation of all of which constitutes the totality of change, which finds the final expression. For instance, the adjective 'good' in the personal context, situated in an emotional narrative between a father and a son, where the father is conveying how good his son is, can be influenced by a family of properties (it can be argued that the worldview encoded in a specific language influences the properties and they can be different for a worldview encoded in a different language) like obedience, politeness, sensibility, moral righteousness, etc., all serving as variables, inevitable time-governed changes which contribute to an inevitable time-governed change in the discursive interpretation of 'good'. Moreover, Van Fraassen vouches that there is no time apart from motion. Motion is not a literal reference to physical movement under the influence of tangible force. Rather, motion suggests interpretative displacement caused by inevitable changes in ideas, events and their interpretations: "Time is said to be a structure of relations used to represent temporal relations, in which all actual temporal relations can necessarily be embedded" (Massey, 1974, p. 91). 
Building up on these philosophical branches of thought, the primary motivation for opening this issue up for discussion, is derived from the compelling work on the temporal structure of discourses being done by the team led by Prof. Martin Becker at the University of Cologne. The focus of the team while investigating tense and aspect in discourse involves "aspectual shaping, temporal anchoring, ordering and perspectivizing of eventualities" (quoted from project website) and the methodological approach involves the use of acceptability tests. While delineating the constitution of a discourse, Becker and Egetenmeyer (2018) elucidate:

[t]he temporal make-up of discourse concerns different "informative levels". First, eventualities are basically ordered as to what happens after what, and what holds while something else happens and suchlike (linear ordering). Second, eventualities are ordered relative to their contribution to a main story line or their belonging to the background. Third, within a text, perspective may be shifted so that eventualities may be viewed from different time points. (p. 28)

Becker and Egetenmeyer stress that all the informative levels are indispensable to understand tense choice in a particular discourse and its role in discourse structuring, specifically while working with narrative texts. It is at this point that the conventional notion of tense choice being the singular tool contributing to a temporal understanding gets debunked by Becker and Egetenmeyer:

tense choice is only one tool contributing to the complexity of the temporal structure in natural discourse. Additionally, not only grammatical and lexical aspect, but also further lexical items such as adverb and conjunctions, and also rhetorical relations play an important role. (2018, pp. 28-29)

\section{Data and findings}

The data collected over 40 native Bangla speaker participants is based on the two following narrative discourses (given with IPA transcriptions and sense translations in English) and their accompanying acceptability tasks with 4 options (given with IPA transcriptions and sense translations in English).

The participants were made to read the following texts labelled as discourse I and II without any constraint on reading time and then select the most acceptable option $(=,<,>$ have usual mathematical implication) from the four that were supplied after the acceptability task question.

P.S. $X_{1}=X_{2}=X_{3}$ would imply that the parameters governing $X$, at time points $t_{1}, t_{2}, t_{3}$, with reference to the discourse timeline, remain unchanged qualitatively. Therefore, the understanding of $X$ would be the same for the referent at $t_{1}, t_{2}, t_{3}$ in the discourse timeline.

$X_{1}>X_{2}>X_{3}$ would imply that the parameters governing $X$, at time points $t_{1}, t_{2}, t_{3}$, with reference to the discourse timeline, show a qualitative decline. Therefore, there will be a depreciation in the understanding of $X$ for the referent across $t_{1}, t_{2}, t_{3}$ in the discourse timeline. 


\section{Discourse I}

অনেক দিন হল বাড়ি ফেরা হয়নি। অনিন্দ্যকে অনেক দিন দেখিনি। অনিন্দ্য ছেলেটা আমার ভালো। ছিল। আজ এতদিন পর বাড়ি ফেরার সুযোগ পেয়ে, সবাইকে দেখার জন্য মন বড় উদগ্রীব হয়ে উঠেছে। কতদিন পর অনিন্দ্যকে আদর করতে পারবো আবার। কোলে হয়তো আর উঠবেনা সে, তবে নতুন জামার বায়না তো করবে। এখনো সে আমার ভালো না, অনিন্দ্যকে যেটুকু সময় কাছে পেয়েছি আমি বুবেছি নে অন্যদের থেকে কতখানি আলাদা। যুগ পাল্টালেও, অনিন্দ্য পাল্টাবে না। আমার ভালো। ছেলে, ভালো

\section{IPA transcription}

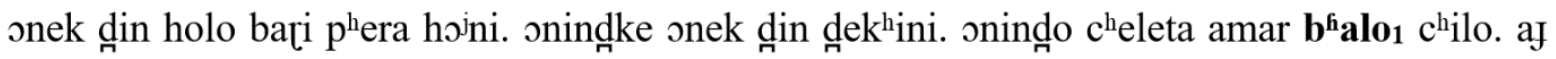

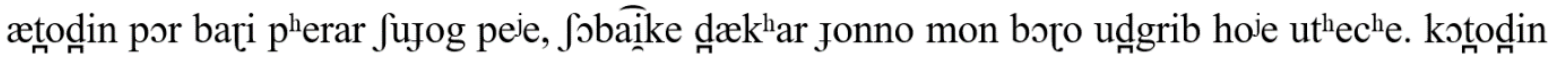

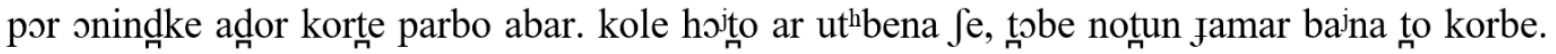

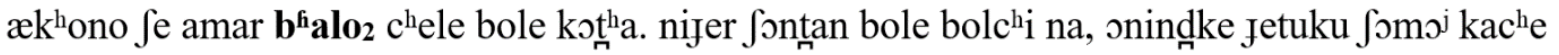

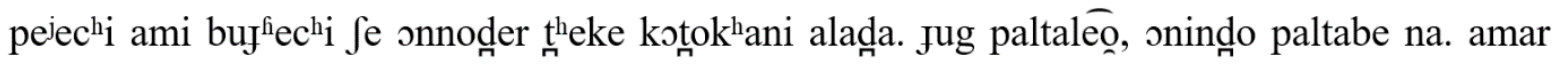

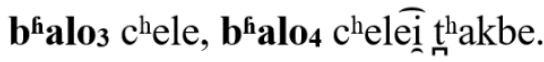

\section{Sense translation in English}

It has been long since my last visit home. I have not seen Anindya for a long time. My son Anindya was a good $\mathbf{~}_{1}$ boy. Getting an opportunity to return home after so long, I feel so restless today to see everyone. After so many days, I will be able to caress Anindya again. He might not want to jump onto my lap but he will certainly demand a new shirt. He is still my $\mathbf{g o o d}_{\mathbf{2}}$ boy. I am not saying this just because Anindya is my son, but whatever time I could spend with him, I realised how different he is from the others. Even if the times change, Anindya will not change. My good $_{3}$ boy will remain a $\mathbf{g o o d}_{4}$ boy.

\section{Options supplied for the acceptability test after reading (self-paced) discourse I}

A. অনিন্দ্য একেবারেই পাল্টায়নি এবং ভবিষ্যতেও পাল্টাবেনা।

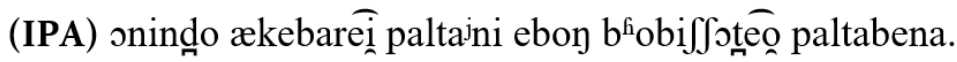

(Sense) Anindya has not changed a bit and will not change in future.

$$
\begin{aligned}
& \text { ভালো }_{1}=\text { ভালো }_{2}=\text { ভালো }_{3}=\text { ভালো }_{4} \\
& \text { b }^{\mathrm{h}} \mathrm{alo}_{1}=\mathrm{b}^{\mathrm{h}} \mathrm{alo}_{2}=\mathrm{b}^{\mathrm{h}} \mathrm{alo}_{3}=\mathrm{b}^{\mathrm{h}} \mathrm{alo}_{4} \\
& \operatorname{good}_{1}=\operatorname{good}_{2}=\operatorname{good}_{3}=\operatorname{good}_{4}
\end{aligned}
$$


13 | Only 'Time' will 'Tell': Influence of temporality on the interpretation of narrative discourses

B. অনিন্দ্যকে তার বাবা যত ভালো দেখে গিয়েছিল, বাবার অনুপস্থিতিতে অনিন্দ্য তার থেকেও বেশি ভালো ছেলে হয়েছে, এবং ভবিষ্যতে সে আরো ভালো হতে পারে।

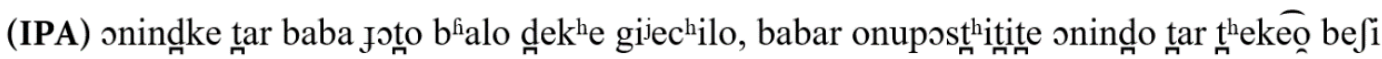

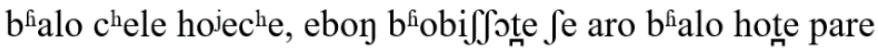

(Sense) The state in which Anindya's father had last seen his son, in his father's absence Anindya has become better as a son and he holds the promise to become even better in future.

$$
\begin{aligned}
& \text { ভালো }\left.\right|_{1}<\text { ভালো }_{2}<\text { ভালো } \\
& \text { b }^{\text {fa }} \text { lo }_{1}<\text { b }^{\text {fialo }} \text { al }_{2}<\text { b }^{\text {ha }} \text { lo }_{4} \\
& \operatorname{good}_{1}<\operatorname{good}_{2}<\operatorname{good}_{4} \\
& \text { C. অনিন্দ্যকে তার বাবা যত ভালো দেখে গিয়েছিল, বাবার অনুপস্থিতিতে এবং সঙ্গদোযে অনিন্দ্য তত ভালো ছেলে আর নেই, যদিও } \\
& \text { এখনো লে অন্যদের তুলনায় ভালো। ভবিষ্যতে সে আরো কম ভালো হতে পারে, আবার বেশি ভালো-ও হতে পারে। }
\end{aligned}
$$

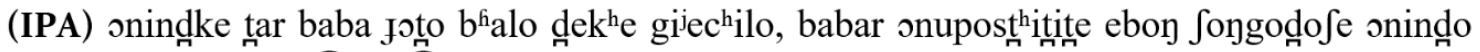

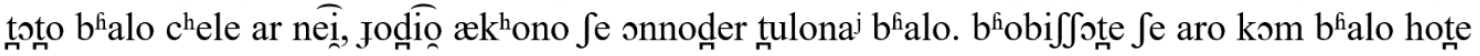
pare, abar be $\int i$ bialo-o hote pare.

(Sense) The state in which Anindya's father had last seen his son, in his father's absence and due to bad company, Anindya is longer that good a boy (or a son), although he still fares better in comparison to the others. In future, he could degrade further, or (now that his father is back) he could improve and become better.

$$
\begin{aligned}
& \text { ভালো }\left.\right|_{1}>\text { ভালো }\left.\right|_{2}>\text { ভালো }\left.\right|_{4} / \text { ভালো }\left.\right|_{1}>\text { ভালো }\left.\right|_{2}<\text { ভালো }
\end{aligned}
$$

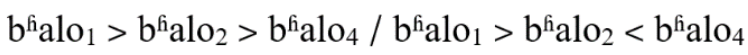

$$
\begin{aligned}
& \operatorname{good}_{1}>\operatorname{good}_{2}>\operatorname{good}_{4} / \operatorname{good}_{1}>\operatorname{good}_{2}<\operatorname{good}_{4} \\
& \text { D. উপরোক্ত তিনটি সম্ভাবনাই প্রযোজ্য। }
\end{aligned}
$$

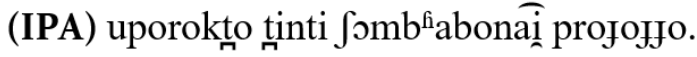

(Sense) All of the three possibilities are applicable.

\section{Discourse II}

পরিবারের সকলের ভালোবাসা আর সমর্থন পেয়ে এসেছে প্রফুল্ল খুব ছোট বয়স থেকেই। ছোটবেলায় সমস্ত খেলাধুলার মধ্যে ফুটবল খেলাটা প্রফুল্ল ভালোবেসে, খেলেছিল। বাড়িতে সবাই নামজাদা ক্রীড়াবিদ। স্বভাবত প্রফুল্লকে নিয়ে সবারই নানা স্বপ্ন ছিল। বড় হয়েও প্রফুল্ল ফুটবলটা ভালোবেসে, খেলতো। ওর উৎসাহ দেখে বাবা- মাও প্রফুল্লকে ফুটবলের দিকেই ঠেলে দিলো। আজ যখন মঞ্চে সে পুরস্কার নিতে উঠবে, প্রফুল্ল হয়তো ফেলে আসা বছরগুলো রোমন্থন করবে না, তবে ফুটবলটা সে ভালোবেসে 


\section{IPA transcription}

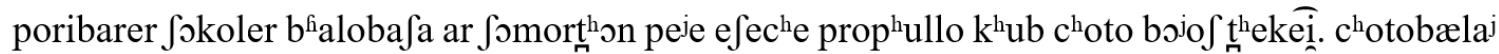

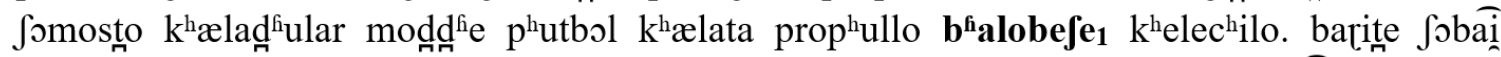

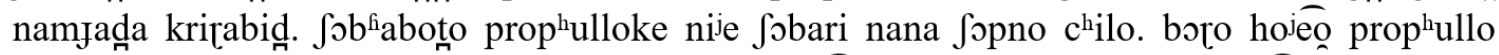

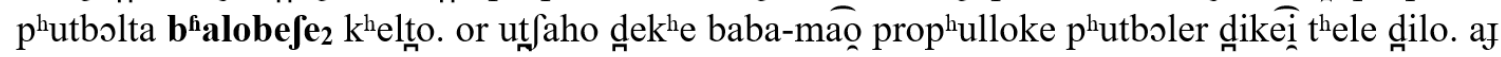

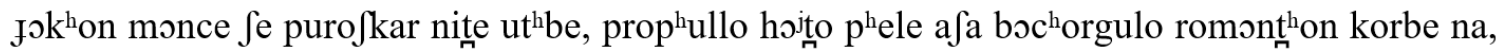
tobe $\mathrm{p}^{\text {h }}$ utbolta $\int \mathrm{e} \mathbf{b}^{\mathrm{f}} \mathbf{a l o b e} \int \mathbf{e}_{3} \mathrm{k}^{\mathrm{h}}$ ele colbe.

\section{Sense translation in English}

Prafulla has received the support and love of all family members from a very young age. In his childhood, out of all games Prafulla played football passionately. Everyone in the family is a renowned sportsperson. Naturally, everyone had dreams surrounding Prafulla. Even after he grew up, Prafulla continued playing football passionately. Witnessing his enthusiasm, his parents pushed Prafulla to take up football professionally. Today, as he will go on stage to collect the trophy, Prafulla might not recollect the years that he has left behind, but he will continue to play football passionately.

\section{Options supplied for the acceptability test after reading (self-paced) discourse II}

\section{A. প্রফুল্লর ফুটবল খেলার প্রতি ভালোবাসা একই রয়েছে এবং থাকবে।}

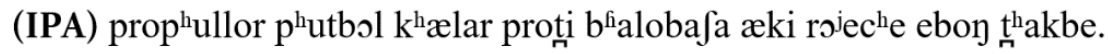

(Sense) Prafulla's passion for the game has remained the same and will be the same.

ভালোবেসে $1=$ ভালোবেসে $2=$ ভালোবেসেস

$b^{\text {ha }}$ alobe $\int e_{1}=b^{\text {ha }}$ alobe $\int e_{2}=b^{\text {hialobe } \int e_{3}}$

passionately $_{1}=$ passionately $_{2}=$ passionately $_{3}$

B. বড় হওয়ার সাথে সাথে এবং খেলোয়াড় হিসেবে সুনাম অর্জন করে, প্রফুল্লর ফুটবলের প্রতি ভালোবাসা বেড়েছে এবং ভবিষ্যতে আরো বাড়বে।

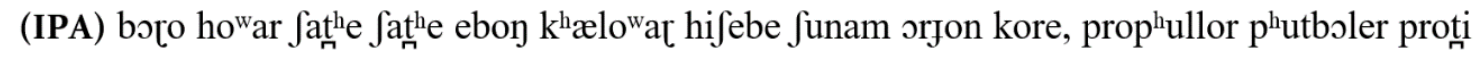

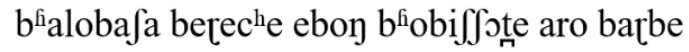

(Sense) In the course of growing up and earning repute as an athlete, Prafulla's passion for football has increased and it will amplify further in the future.

$$
\begin{aligned}
& \text { ভালোবেসে }
\end{aligned}
$$

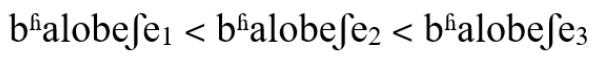

$$
\begin{aligned}
& \text { passionately }_{1}<\text { passionately }_{2}<\text { passionately }_{3}
\end{aligned}
$$


15 | Only 'Time' will 'Tell': Influence of temporality on the interpretation of narrative discourses

C. ছোটবেলার উৎসাহ স্বাভাবিকভাবেই বয়স বাড়ার সাথে সাথে প্রফুল্লর মধ্যে নেই; প্রফুল্লর ফুটবলের প্রতি ভালোবাসা সামান্য হলেও কমেছে এবং ভবিষ্যতে শারীরিক ক্ষমতা হ্রাসের সাথে খেলার প্রতি ভালোবাসা আরো কমতে পারে।

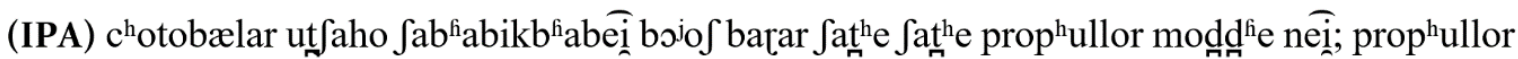

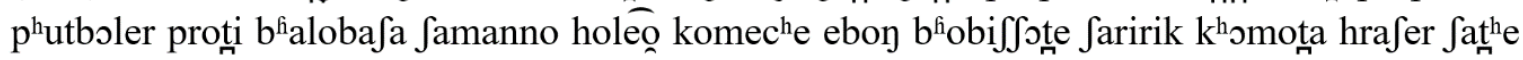
$\mathrm{k}^{\mathrm{h}} \mathfrak{\text { lar }}$ proti $\mathrm{b}^{\mathrm{h}}$ aloba $\int \mathrm{a}$ aro komte pare.

(Sense) The childhood passion is naturally not there anymore as Prafulla has grown in age; Prafulla's passion for football has decreased even if it's by a tiny bit and in future it will decrease further with loss of physical strength.
ভালোবেসে $>$ > ভালোবেসে 2 > ভালোবেসেস 3
b ${ }^{\text {halobe } \int e_{1}}>$ b $^{\text {halobe }} \int \mathrm{e}_{2}>$ b $^{\text {halobe }} \int \mathrm{e}_{3}$
passionately $_{1}>$ passionately $_{2}>$ passionately $_{3}$
D. উপরোক্ত তিনটি সম্ভাবনাই প্রযোজ্য।

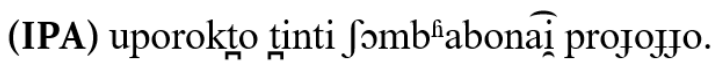

(Sense) All of the three possibilities are applicable.

\section{Participant responses}

\begin{tabular}{|l|l|l|}
\hline $\begin{array}{l}\text { Participant } \\
\text { Code }\end{array}$ & $\begin{array}{l}\text { Response to acceptability test after } \\
\text { analysing discourse I } \\
\text { (adjective focused) }\end{array}$ & $\begin{array}{l}\text { Response to acceptability test after } \\
\text { analysing discourse II } \\
\text { (adverb focused) }\end{array}$ \\
\hline P1 & D & D \\
\hline P2 & C & A \\
\hline P3 & B & B \\
\hline P4 & B & B \\
\hline P5 & D & D \\
\hline P6 & A & B \\
\hline P7 & A & B \\
\hline P8 & C/D & C \\
\hline P9 & A & C \\
\hline P10 & A & C \\
\hline P11 & C(2) & C \\
\hline P12 & D & D \\
\hline P13 & A & B \\
\hline P14 & B & B \\
\hline P15 & D & A \\
\hline P16 & D & D \\
\hline P17 & B & C \\
\hline & & \\
\hline
\end{tabular}




\begin{tabular}{|l|l|l|}
\hline P18 & D & B \\
\hline P19 & D & D \\
\hline P20 & B & C \\
\hline P21 & D & B \\
\hline P22 & A & B \\
\hline P23 & D & D \\
\hline P24 & C & A \\
\hline P25 & D & C \\
\hline P26 & A & B \\
\hline P27 & B & D \\
\hline P28 & D & B \\
\hline P29 & B & C \\
\hline P30 & D & D \\
\hline P31 & A & B \\
\hline P32 & B & B \\
\hline P33 & D & B \\
\hline P34 & B & D \\
\hline P35 & D & A \\
\hline P36 & A & C \\
\hline P37 & C & B \\
\hline P38 & A & C \\
\hline P39 & B & C \\
\hline P40 & C & D \\
\hline & A & \\
\hline
\end{tabular}

Table 1: Acceptability task results tabulated after anonymising participants as per their consent
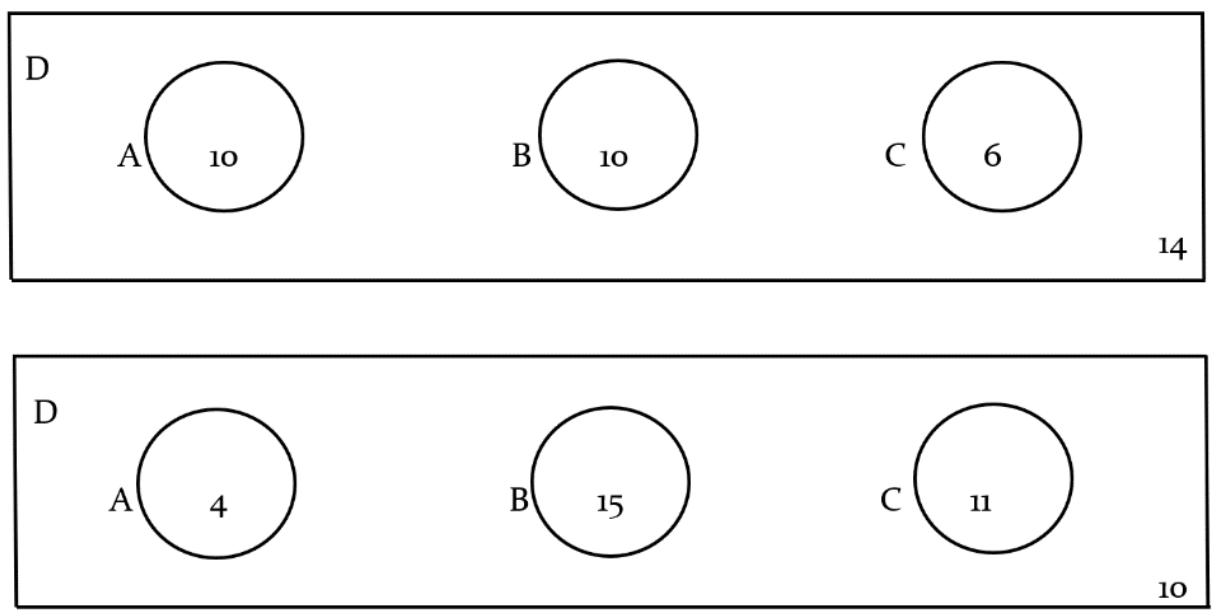

Table 2: Venn diagram representation of proportion of participant choices

\begin{tabular}{|l|l|l|}
\hline & Time-independent readings (A) & Time dependent readings (B \& C) \\
\hline $\begin{array}{l}\text { Discourse I } \\
\text { (adjective focused) }\end{array}$ & $24(35 \%)$ & $44(65 \%)$ \\
\hline
\end{tabular}


17 | Only 'Time' will 'Tell': Influence of temporality on the interpretation of narrative discourses

\begin{tabular}{|l|l|l|}
\hline $\begin{array}{l}\text { Discourse II } \\
\text { (adverb focused) }\end{array}$ & $14(23 \%)$ & $46(77 \%)$ \\
\hline
\end{tabular}

Table 3: Percentage of time-independent versus dependent readings

\section{Inference}

The participant choices in the acceptability test task strongly favour a time-derived interpretation of the focused adjective or the focused adverb, as the participant encounters its repetitions at different points of time in the discourse timeline.

Both discourses I and II are personal narratives in the familial context with a strong

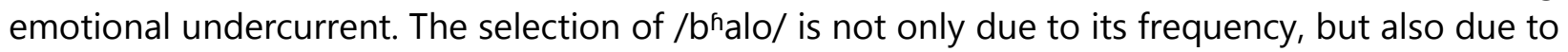
the fact that its interpretation is cumulatively reinforced right from a very young age, being tied to lessons on virtue, obedience in language primers and then moving to karmic implications. This can possibly justify the 10 time-independent readings that the acceptability test generates on the option A, which records a static interpretation.

The numbers do open an interesting avenue of possibilities that the ongoing doctoral dissertation, of which this paper is a small part, explores convincingly. The Bangla adjective /bhalo/

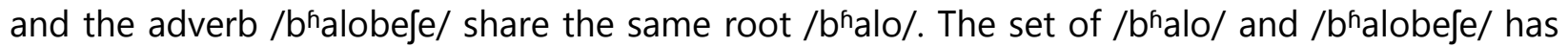
been planned with the objective of sharing a root, which will in turn add a contrastive dimension to the study, i.e., when adjectives and adverbs are formed from the same root, how strong or weak are the discourse-time-derived readings. Evidently, as per the findings, the discourse-time-derived readings are somewhat stronger for the adverb (approx. 77\%) when compared to the adjective (approx. 65\%) sharing the same root (/bhalo/).

\section{Methodology}

The general methodological standpoint for the paper is that of discourse analysis. However, to be absolutely precise, the approach of $Q$-methodology is adopted to analyse how language users interpret certain focused expressions/ideas within the temporal structure of narrative discourses.

Q-Methodology is the most apt, as it deals with the self-interpretations of individuals. In Q-Methodology, the participants are confronted with a problem-solving exercise probing their self-understandings, which is the acceptability test with the four options, the last being the same for all, i.e. agreeing with the acceptability of the first three options collectively. As Glynos (2009) puts it, "Q methodology emphasizes the active role of subjects in the generation and appropriation of meaning, but offers a quantitative way to make this sort of research systematic and its findings as robust and reliable as possible" (p. 28).

The approach positively exploits the interpretive capacities of participants, with respect to the issue being examined, in a two-fold manner:

First, it pushes participants into a situation where a decision is needed as to what is "meaningful" and thereby what is (and what is not) of relevance and importance from the perspective of the participants (Watts \& Stenner, 2005). 
Second, the self-interpretations gathered from the participants, are then "subjected to interpretation by the researchers, thereby yielding a form of contextualized self-interpretation" (Glynos, 2009, p. 29).

\section{Funding Disclosure/Acknowledgement}

This paper is part of an ongoing doctoral dissertation at the Centre for Linguistics, School of Language, Literature \& Culture Studies, Jawaharlal Nehru University. A major part of this work was done under the Research Fellowship Program in Sciences, Humanities \& Social Sciences of the University Grants Commission, India. The researcher was a Senior Research Fellow from July 2017 to July 2020.

\section{References}

Ardley, G. (1968). Berkeley's Renovation of Philosophy. Martinus Nijoff.

Augustine, S. (1963). The Confessions (R. Warner, Trans.). Mentor-Omega Books.

Becker, M., \& Egetenmeyer, J. (2018). A prominence-based account of temporal discourse structure. Lingua, 214, 28-58. https://doi.org/10.1016/j.lingua.2018.08.002

Becker, M., \& Donazzan, M. (2017). Or, ora, maintenant: perspective temporelle et perspective argumentative. Discours. Revue de linguistique, psycholinguistique et informatique. A journal of linguistics, psycholinguistics and computational linguistics, 20. https://doi.org/ $10.4000 /$ discours.9290

Berkeley, G. (1881). A treatise concerning the principles of human knowledge. JB Lippincott \& Company.

Berkeley, G., \& Luce, A. A. (1944). Philosophical Commentaries; Generally Called the Commonplace Book. Thomas Nelson \& Sons Ltd.

Berkeley, G. (1949). The Principles of Human Knowledge. In A. A. Luce \& T. E. Jessop (Eds.), The Works of George Berkeley, Bishop of Cloyne (Vol. 2) (pp. 1-114). Thomas Nelson \& Sons Ltd.

Farkas, K. (2008). Time, tense, truth. Synthese, 160(2), 269-84. https://doi.org/10.1007/s11229-006-9116-y

Garber, D. (1982). Locke, Berkeley, and Corpuscular Scepticism. In C. M. Turbayne (Ed.), Berkeley: Critical and Interpretive Essays (pp. 174-196). University of Minnesota Press.

Glynos, J., Howarth, D., Norval, A. \& Speed, E. (2009). Discourse analysis: Varieties and methods. ESRC National Centre for Research Methods Networks for Methodological Innovation. Retrieved March 5, 2019, from https://www.researchgate.net/publication/279668539_Discourse_Analysis_varieties_and_methods

Grayling, A. C. (1986). Berkeley: The Central Arguments. Gerald Duckworth and Co. Ltd.

Helm, B. P. (1980). The nature and modes of time. The Monist, 63(3), 375-385. https://doi.org/10.5840/monist198063324

Hynes, D. (2005). Berkeley's Corpuscular Philosophy of Time. History of Philosophy Quarterly, 22(4), 33956. https://www.jstor.org/stable/27745036 
Kant, I. (1998). Critique of Pure Reason (P. Guyer \& A. W. Wood, Trans.). Cambridge University Press.

Kneebone, G. T. (1964). [Review of the book The Natural Philosophy of Time, by G. J. Whitrow]. Philosophy, 39(147), 86-88. https://www.jstor.org/stable/3749141

Lacey, H. M. (1968). Empiricism and Augustine's Problems about Time. The Review of Metaphysics, 22(2), 219-245. https://www.jstor.org/stable/20124811

Locke, J. (1860). An essay concerning human understanding. And a treatise on the conduct of the understanding. Complete in one volume: with the author's last additions and corrections. Hayes \& Zell.

Massey, G. J. (1974). [Review of the book An Introduction to the Philosophy of Time and Space, by B. C. van Fraassen]. Philosophy of Science, 41(1), 90-92. https://www.jstor.org/stable/187381

McTaggart, J. McT. E. (1927). The Nature of Existence (Vol. 2) (C. D. Broad, Ed.). Cambridge University Press.

Peirce, C. S. (1974). Collected Papers of Charles Sanders Peirce (Vol. 2) (C. Hartshorne \& P. Weiss, Eds.). Harvard University Press.

Pitcher, G. (1977). Berkeley. Routledge and Kegan Paul.

Tipton, I. C. (1977). Berkeley: The Philosophy of Immaterialism. Methuen and Co. Ltd.

Van Fraassen, B. C. (1970). An Introduction to the Philosophy of Time and Space. Columbia University Press.

Watts, S., \& Stenner, P. (2005). Doing Q methodology: theory, method and interpretation. Qualitative research in psychology, 2(1), 67-91. https://doi.org/10.1191/1478088705qp022oa

Whitrow, G. J. (1961). The Natural Philosophy of Time. Thomas Nelson and Sons Ltd.

Worms, F. (2005). Time thinking: Bergson's double philosophy of mind. MLN, 120(5), 1226-1234. https://doi.org/10.1353/mln.2006.0025

Zwart, P. J. (1973). The flow of time. In P. Suppes (Ed.), Space, Time and Geometry (pp. 131-156). Springer. https://doi.org/10.1007/978-94-010-2686-4

Bate, J. (1999). Shakespeare and original genius. In P. Murray (Ed.), Genius - The History of an Idea. New York: Basil Blackwell. 76-97

Debmalya Biswas is a final-year Doctoral Researcher at the Centre for Linguistics, Jawaharlal Nehru University, having interest in Discourse Analysis, Philosophy of Language, Sociolinguistics and ELT. He has been a Course Editor and Course Writer for the School of Interdisciplinary and Transdisciplinary Studies (SOITS) and the School of Humanities, IGNOU, New Delhi and for the School of Humanities, NSOU, Kolkata. He has also been a Resource Person and Subject Matter Expert for the Annual Refresher Programme in English Language Teaching on SWAYAM offered by the National Resource Centre for ELT, UGC-HRDC, Gujarat University. 